\title{
Insulin resistance induced by sucrose feeding in rats is due to an impairment of the hepatic parasympathetic nerves
}

Received: 30 September 2004 / Accepted: 12 December 2004 / Published online: 14 April 2005

(C) Springer-Verlag 2005

\begin{abstract}
Aims/hypothesis: A considerable proportion of whole-body insulin-stimulated glucose uptake is dependent upon the hepatic insulin-sensitising substance (HISS) in a pathway mediated by the hepatic parasympathetic nerves (HPNs). We tested the hypothesis that a high-sucrose diet leads to the impairment of the HPN-dependent component of insulin action. Methods: We quantified insulin sensitivity using the rapid insulin sensitivity test, a modified euglycaemic clamp. Quantification of the HPN-dependent component was achieved by administration of a muscarinic receptor antagonist (atropine, $3 \mathrm{mg} / \mathrm{kg}$ ). Results: Insulin sensitivity was higher in standard-fed than in sucrose-fed Wistar rats $(305.6 \pm 34.1 \mathrm{vs} 193.9 \pm 13.7 \mathrm{mg}$ glucose $/ \mathrm{kg}$ body weight; $p<0.005)$ and Sprague-Dawley rats $(196.4 \pm 5.9$ vs $95.5 \pm 16.3 \mathrm{mg}$ glucose $/ \mathrm{kg}$ body weight; $p<0.01$ ). The HPNindependent component was similar in the two diet groups. Insulin resistance was entirely due to an impairment of the HPN-dependent component in both Wistar rats $(164.3 \pm 28.1$ [standard-fed] vs 26.5 \pm 7.5 [sucrose-fed] $\mathrm{mg}$ glucose $/ \mathrm{kg}$ body weight; $p<0.0001)$ and Sprague-Dawley rats $(111.7 \pm$ 9.5 vs $35.3 \pm 21.4 \mathrm{mg}$ glucose $/ \mathrm{kg}$ body weight; $p<0.01$ ). Furthermore, HPN-dependent insulin resistance in Sprague-
\end{abstract}

R. T. Ribeiro - M. P. Macedo ( $₫)$

Department of Physiology, Faculty of Medical Sciences,

New University of Lisbon,

Campo Mártires da Pátria, 130,

1169-056 Lisbon, Portugal

e-mail: mpmacedo.biot@,fcm.unl.pt

Tel.: +351-21-8803017

Fax: +351-21-8803023

W. W. Lautt · D. J. Legare

Department of Pharmacology and Therapeutics,

Faculty of Medicine, University of Manitoba,

Winnipeg, MB, Canada

W. W. Lautt

Canadian Institutes for Health Research,

Ottawa, Canada

M. P. Macedo

Portuguese Diabetes Association,

Lisbon, Portugal
Dawley rats was already evident after 2 weeks of a highsucrose $\operatorname{diet}(28.5 \pm 7.6$ [2 weeks], 35.3 \pm 21.4 [6 weeks], $17.9 \pm$ 5.4 [9 weeks] $\mathrm{mg}$ glucose $/ \mathrm{kg}$ body weight) and was in-

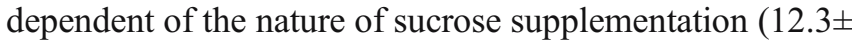
4.7 [solid] and 17.9 \pm 5.4 [liquid] $\mathrm{mg}$ glucose $/ \mathrm{kg}$ body weight). Conclusions/interpretation: Our results support the hypothesis that insulin resistance caused by sucrose feeding is due to an impairment of the HPN-dependent component of insulin action, leading to a dysfunction of the HISS pathway.

Keywords Hepatic insulin-sensitising substance $\cdot$ Hepatic parasympathetic nerves $\cdot$ Insulin resistance $\cdot$ Skeletal muscle $\cdot$ Sucrose $\cdot$ Type 2 diabetes mellitus

Abbreviations HISS: Hepatic insulin-sensitising substance - HPN: Hepatic parasympathetic nerve - RIST: Rapid insulin sensitivity test · SD: Sprague-Dawley · WIS: Wistar

\section{Introduction}

The hepatic parasympathetic nerves (HPNs) are essential regulators of the normal action of insulin on glucose metabolism. The activation of the HPNs during the immediate postprandial state leads to the release, by the liver, of a putative hormone referred to as hepatic insulin-sensitising substance (HISS). The HISS hypothesis states that HISS travels through the bloodstream to enhance skeletal muscle insulin-stimulated glucose uptake, consequently accounting for $50-60 \%$ of whole-body glucose uptake [1].

The parasympathetic neural reflex that leads to HISS release is conducted via the cervical vagus, the hepatic branch of the vagus and the anterior hepatic plexus. The decrease in insulin sensitivity obtained with HPN denervation is also achieved pharmacologically, by administration of the muscarinic antagonist atropine [2]. Parasympathetic denervation of the other splanchnic organs, or sympathetic denervation of the liver, does not alter insulin sensitivity [3]. The fact that acetylcholine, when administered intraportally 
but not intravenously, was able to reverse the insulin resistance induced by surgical intervention, further established the liver as the target organ $[4,5]$. Although this could lead us to consider that the liver becomes insulin resistant, studies of arterial-venous gradients showed that it is mainly the skeletal muscle that develops insulin resistance. While glucose uptake by the skeletal muscle decreases, insulin retains its capacity to inhibit glucose output from the liver $[4,5]$. Furthermore, the existence of a hepatic blood-borne factor that increases skeletal muscle glucose uptake had already been hinted in cases of liver disease [6]. Chronic liver disease also presents a predominance of parasympathetic tone dysfunction related to the severity of disease [7-9].

Parasympathetic dysfunction has been associated with severe insulin resistance [10], which predates the overt development of type 2 diabetes mellitus by many years [11]. The impairment of the parasympathetic nervous system has recently been considered as an aetiological factor in the pathological process, rather than just a consequence of diabetes [12].

It is well established that sucrose feeding, which is increasing in human diet, leads to the development of skeletal muscle insulin resistance [13-16]. However, until now, the importance of the HPNs has not been assessed in studies of insulin resistance induced by sucrose feeding.

Since it has been shown that differences in strain characteristics between Wistar and Sprague-Dawley rats can lead to variations in whole-body insulin sensitivity [17], these two strains, the most commonly used for insulinstimulated glucose measurements, were used in this study.

We aimed to relate the insulin resistance caused by a highsucrose diet to the HISS hypothesis, a pathway dependent upon the HPN modulation of whole-body insulin-stimulated glucose uptake.

\section{Materials and methods}

Animals

Male Wistar (WIS) and Sprague-Dawley (SD) rats were obtained from Charles River Laboratories (Barcelona, Spain). After weaning, they were maintained on a 12-h light-dark cycle (08.00-20.00 hours) and housed one per cage under temperature control. All rats were fed ad libitum, except on the day before the experiment when they started an overnight fast. Animals were cared for according to the European Union Directive for Protection of Vertebrates Used for Experimental and Other Scientific Ends (86/609/ CEE), the US National Research Council Guide for the Care and Use of Laboratory Animals and the Canadian Council on Animal Care.

\section{Diet protocols}

Effect of a liquid high-sucrose diet on Wistar and SpragueDawley rats WIS and SD rats were each randomly divided into two groups. Both standard-fed and sucrose-fed rats had free access, for 6 weeks, to a standard solid diet (Panlab A04; Charles River) and water, but while the standard-fed groups had only a bottle of drinking water, the sucrose-fed groups had an additional bottle of a $35 \%$ sucrose solution.

Effect of the duration of exposure to a liquid high-sucrose diet in Sprague-Dawley rats SD rats were randomly divided into six groups. Each sucrose-fed group was given a standard solid diet (Panlab A04; Charles River) and a 35\% sucrose solution for, respectively, 2, 6 or 9 weeks. Each standard-fed group was given a standard solid diet and drinking water for time periods corresponding to the sucrose-fed groups.

Effect of a liquid vs solid high-sucrose diet in SpragueDawley rats SD rats were randomly divided into three groups. The standard-fed group was given a standard solid diet (Research Diets, Brunswick, NJ, USA) and drinking water for 9 weeks. Sucrose-fed groups were given either a $35 \%$ sucrose solid diet (Research Diets) and drinking water or a standard solid diet and a 35\% sucrose solution for the same period.

\section{Presurgical protocol}

Rats were fasted overnight and allowed access to food for 1 h (08.00-09.00 hours). They were anaesthetised with an intraperitoneal injection of sodium pentobarbital $(65 \mathrm{mg} / \mathrm{kg})$ and were placed on a heating pad (Homeothermic Blanket Control Unit 50-7061; Harvard Apparatus, Holliston, MA, USA) to maintain body temperature at $37.5 \pm 0.5^{\circ} \mathrm{C}$, monitored with a rectal probe thermometer.

\section{Surgical protocol}

The trachea was cannulated (polyethylene tubing, PE 240; Becton Dickinson, Franklin Lakes, NJ, USA) to allow for spontaneous respiration. The femoral artery and the jugular vein were cannulated (polyethylene tubing, PE 50, Becton Dickinson) to establish an external arterial-venous shunt, primed with a saline-heparin solution $(200 \mathrm{U} / \mathrm{ml})$. This shunt also enabled the periodic measurement of the arterial and venous pressures (Powerlab 8/s, ADInstruments; recorded by MacLab software, Colorado Springs, CO, USA). Anaesthesia was maintained throughout the experiment by continuous infusion of sodium pentobarbital solution (1.0 $\mathrm{mg} / \mathrm{ml}$ saline given at $1.0 \mathrm{ml} / 100 \mathrm{~g}$ body weight) through a cannula (infusion line PE 50 with a cut 23 -g needle at the delivery end) inserted into the venous side of the shunt. All drug and saline solutions were administered i.v. through this shunt.

The rats were allowed to stabilise after surgery for at least $30 \mathrm{~min}$ before any tests were carried out. After that time, arterial blood samples $(25 \mu \mathrm{l})$ were taken from the shunt every $5 \mathrm{~min}$ and the glucose concentration was immediately determined by the oxidase method using a glucose analyser (1500 Sidekick; Yellow Springs Instruments, 
Yellow Springs, OH, USA) until three successive stable glucose concentrations were obtained. The mean of these three values is referred to as the basal glucose level.

Rapid insulin sensitivity test

Minute 0 was set at the start of a 5 -min i.v. insulin bolus ( 50 $\mathrm{mU} / \mathrm{kg}$ ) given using a perfusion pump (Perfusor fm; BBraun Medical, Barcarena, Portugal). At minute 1, the arterial blood glucose concentration was measured and a glucose infusion (D-glucose/saline, $100 \mathrm{mg} / \mathrm{ml}$, i.v.) was started at a rate of $5 \mathrm{mg} \cdot \mathrm{kg}^{-1} \cdot \mathrm{min}^{-1}$ to avoid hypoglycaemia. Arterial blood glucose concentration was measured at 2-min intervals and the rate of the glucose infusion was adjusted whenever necessary to maintain the glycaemia as near as possible to the basal glucose level. The rapid insulin sensitivity test (RIST) was concluded when no further glucose was required. The amount of glucose infused quantifies insulin sensitivity and is referred to as the RIST index (mg glucose/kg body weight).

\section{Experimental protocol for HPN blockade}

After the control RIST was performed, atropine ( $3 \mathrm{mg} / \mathrm{kg}$ ) was infused i.v. into the arterial-venous shunt over $5 \mathrm{~min}$. Glucose levels were allowed to stabilise for at least $30 \mathrm{~min}$, after which another RIST was performed.

\section{Calculations}

The RIST index obtained after atropine-induced HPN blockade represents the HPN-independent component of insulin action. By subtracting the RIST index obtained after pharmacological intervention from the control RIST, one quantifies the HPN-dependent component of insulin action.

To obtain the curves representing the time-course of each RIST we plotted the mean values of glucose infusion rate, represented at 0.1-min intervals. The HPN-dependent component action curve was obtained by subtracting the postatropine curve values from the corresponding control curve values.

\section{Data analysis}

Data were expressed as means $\pm \mathrm{SE}$ and were analysed by unpaired $t$-test or one-way ANOVA followed by the Tukey multiple-comparison test. Differences were accepted as statistically significant if $p$ was less than 0.05 .

\section{Drugs}

Atropine and D-glucose were purchased from SigmaAldrich (Sintra, Portugal). Sodium pentobarbital (Eutasil) was obtained from Ceva (Algés, Portugal). Heparin was purchased from BBraun Medical. Human insulin (Humulin regular) was obtained from Lilly Farma (Algés, Portugal). All chemicals were dissolved in saline (BBraun). All perfusions were carried out using perfusor pumps (BBraun).

\section{Results}

Effect of a liquid high-sucrose diet on Wistar and SpragueDawley rats There were no significant differences in total body weight, blood pressure and postprandial basal glycaemia between the standard-fed $(n=8)$ and sucrose-fed $(n=10)$ WIS rats or between the standard-fed $(n=15)$ and sucrose-fed $(n=5)$ SD rats (data not shown).

In WIS rats, the standard-fed group had higher total insulin sensitivity than the sucrose-fed group (305.6 434.1 [standard-fed] vs 193.9 \pm 13.7 [sucrose-fed] mg glucose $/ \mathrm{kg}$ body weight; $p<0.005$ ). The RIST indexes obtained after atropine administration, which measure the HPN-independent component of insulin action, were similar in the two groups (140.4 \pm 22.1 [standard-fed] and 167.4 \pm 10.8 [sucrosefed] mg glucose/kg body weight) (Fig. 1).

The HPN-dependent component of insulin action was significantly lower in the sucrose-fed than in the standardfed group (164.3 \pm 28.1 [standard-fed] vs $26.5 \pm 7.5$ [sucrosefed] $\mathrm{mg}$ glucose $/ \mathrm{kg}$ body weight; $p<0.0001$ ). This means that the contribution of the HPN-dependent component to total insulin action was diminished in sucrose-fed WIS rats $(52.1 \pm 6.6 \%$ [standard-fed] vs $12.7 \pm 3.3 \%$ [sucrosefed]; $p<0.0001)$.

The dynamic profiles of the RISTs obtained before and after atropine administration and the dynamic profile of the HPN-dependent component of WIS rats are displayed (Fig. 2) and their characteristics described (Table 1).

The standard-fed SD rats also had higher total insulin sensitivity than the sucrose-fed SD rats $(196.4 \pm 5.9$ [standard-fed] vs $95.5 \pm 16.3$ [sucrose-fed] $\mathrm{mg}$ glucose $/ \mathrm{kg}$ body weight; $p<0.01$ ). Since the HPN-independent component

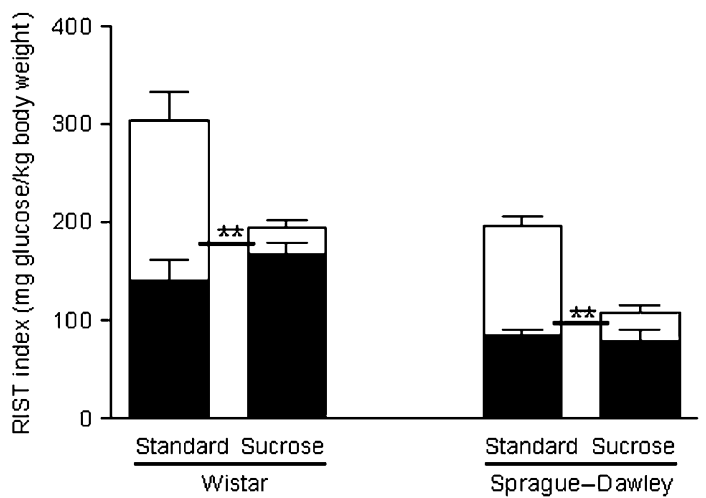

Fig. 1 RIST indexes for the HPN-independent (filled) and HPNdependent (blank) components of insulin action on Wistar and Sprague-Dawley rats. The sum of both components represents the control RIST index for each diet protocol. In both strains, insulin sensitivity was significantly higher for the standard-fed diet group than for the high-sucrose-fed group $(* * p<0.01)$. The HPN-dependent component accounted for this difference 


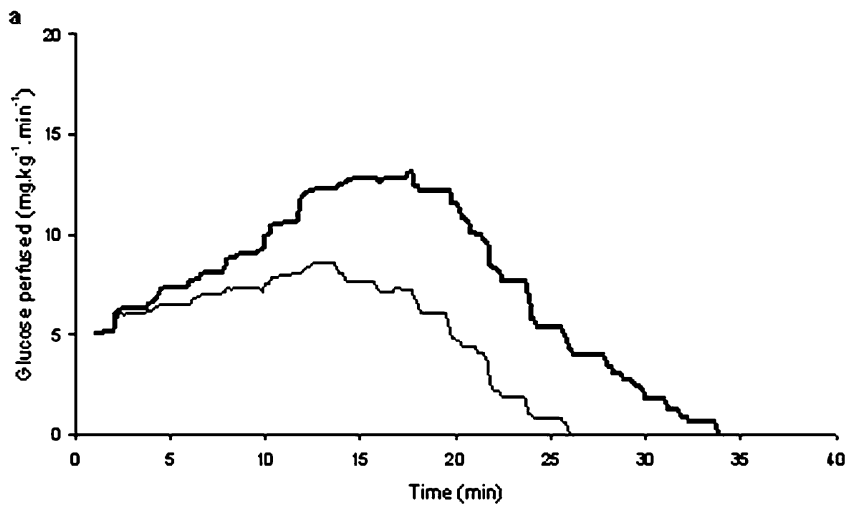

b
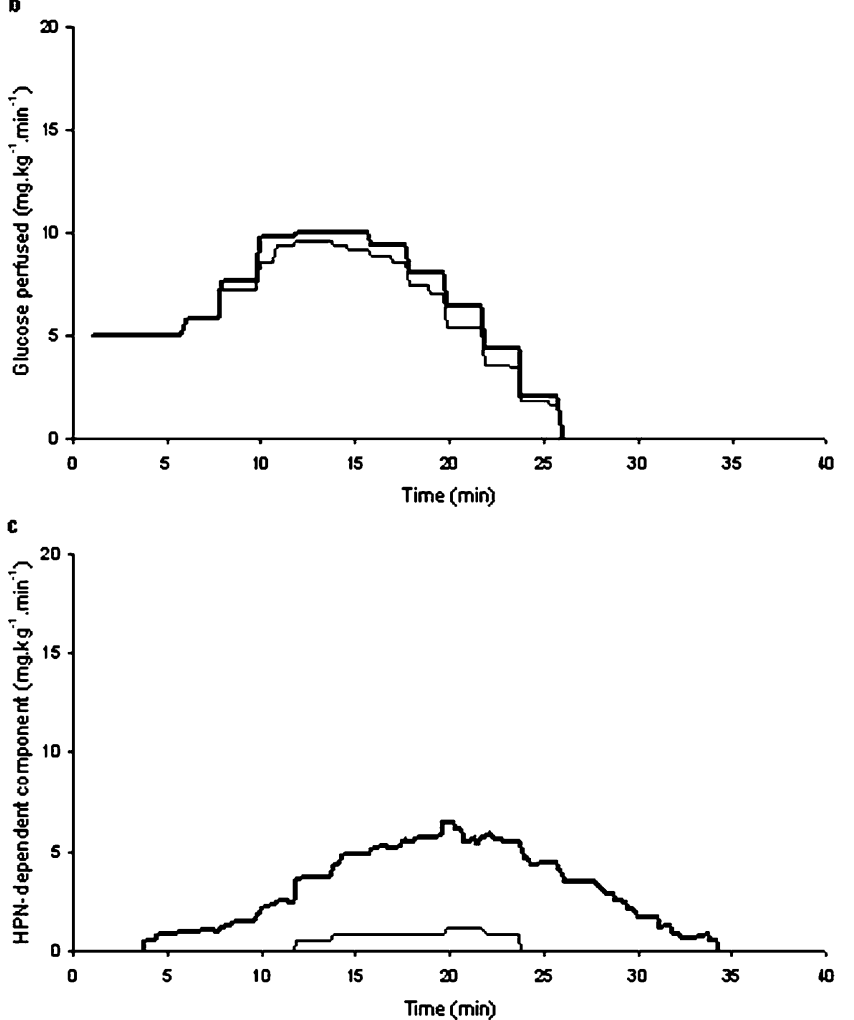

Fig. 2 Dynamic profiles of the control (bold line) and post-atropine (thin line) RISTs of standard-fed (a) and high-sucrose-fed (b) Wistar rats. c Dynamic profile of the HPN-dependent component of insulin action, obtained by subtracting the post-atropine curve from the control curve, of the standard-fed (bold line) and high-sucrose-fed (thin line) Wistar rats

was similar with both diets $(84.7 \pm 6.0$ [standard-fed] and $61.0 \pm 10.1$ [sucrose-fed] $\mathrm{mg}$ glucose $/ \mathrm{kg}$ body weight), the reduced insulin action observed in sucrose-fed SD rats was due to a decrease of the HPN-dependent component (111.7 \pm 9.5 [standard-fed] vs $35.3 \pm 21.4$ [sucrose-fed] $\mathrm{mg}$ glucose/ $\mathrm{kg}$ body weight; $p<0.01$ ). Accordingly, the contribution of the HPN-dependent component to the total insulin sensitivity was lower for the sucrose-fed group $(56.0 \pm 3.7 \%$ [standard-fed] vs $28.8 \pm 13.8 \%$ [sucrose-fed]; $p<0.05)$.

Although the absolute values were lower in SD than in WIS rats, the contribution of the HPN-dependent component was similar in the two strains for both the standard-fed and sucrose-fed groups. Also, the dynamic profiles obtained
Table 1 Dynamic profile characteristics of the control RIST, the HPN-independent component (post-atropine RIST), and the HPNdependent component of total insulin action

\begin{tabular}{|c|c|c|c|}
\hline & & Standard-fed & Sucrose-fed \\
\hline \multirow[t]{3}{*}{ Control RIST } & $\begin{array}{l}\text { Peak (mg glucose } \\
\left.\mathrm{kg}^{-1} \cdot \mathrm{min}^{-1}\right)\end{array}$ & $15.9 \pm 0.7^{\mathrm{a}}$ & $11.7 \pm 1.3^{\mathrm{a}}$ \\
\hline & Peak time (min) & $12.3 \pm 0.5$ & $12.6 \pm 0.8$ \\
\hline & Offset (min) & $35.8 \pm 1.8^{\mathrm{a}}$ & $26.8 \pm 0.5^{\mathrm{a}}$ \\
\hline \multirow[t]{3}{*}{$\begin{array}{l}\text { HPN-independent } \\
\text { component }\end{array}$} & $\begin{array}{l}\text { Peak (mg glucose } \\
\left.\mathrm{kg}^{-1} \cdot \mathrm{min}^{-1}\right)\end{array}$ & $10.8 \pm 1.1$ & $10.2 \pm 1.2$ \\
\hline & Peak time (min) & $11.1 \pm 0.8$ & $11.8 \pm 0.9$ \\
\hline & Offset (min) & $24.9 \pm 2.1$ & $26.8 \pm 0.5$ \\
\hline \multirow{5}{*}{$\begin{array}{l}\text { HPN-dependent } \\
\text { component }\end{array}$} & Onset (min) & $7.2 \pm 1.0$ & $8.9 \pm 1.7$ \\
\hline & $\begin{array}{l}\text { Peak (mg glucose } \\
\left.\mathrm{kg}^{-1} \cdot \min ^{-1}\right)\end{array}$ & $10.0 \pm 1.1^{\mathrm{b}}$ & $1.5 \pm 0.6^{\mathrm{b}}$ \\
\hline & Peak time (min) & $15.7 \pm 1.1$ & $16.1 \pm 1.9$ \\
\hline & Offset (min) & $35.4 \pm 1.9^{\mathrm{a}}$ & $22.9 \pm 2.1^{\mathrm{a}}$ \\
\hline & Duration (min) & $26.6 \pm 2.1^{\mathrm{a}}$ & $8.3 \pm 2.0^{\mathrm{a}}$ \\
\hline
\end{tabular}

for SD rats were similarly shaped to the control RIST, postatropine RIST and HPN-dependent component of WIS rats.

Effect of the duration of exposure to a liquid high-sucrose diet in Sprague-Dawley rats While blood pressure (data not shown) was similar in all standard-fed and sucrose-fed groups, the sucrose-fed group showed, at 9 weeks of sucrose supplementation and in comparison with the standard-fed group, higher body weight $(459.1 \pm 4.7 \mathrm{~g}$ [standard-fed] vs $643.1 \pm 25.9 \mathrm{~g}$ [sucrose-fed]; $p<0.0001)$ and higher basal glycaemia (5.91 \pm 0.18 [standard-fed] vs $7.91 \pm 0.49$ [sucrosefed] mmol glucose $/ 1 ; p<0.05$ ). For all other exposure times, these parameters were similar in sucrose-fed and standardfed rats.

Since all standard-fed groups had similar values of control and post-atropine RIST indexes, we pooled them into a single standard-fed group ( $n=24)$ (Fig. 3). All sucrose-fed groups had similar total insulin sensitivity $(106.2 \pm 12.5$ [2 weeks, $n=7$ ], $95.5 \pm 16.3$ [ 6 weeks, $n=5$ ] and 106.6 \pm 8.4 [9 weeks, $n=11] \mathrm{mg}$ glucose/kg body weight), which was lower than in the standard-fed pool $(192.4 \pm 4.9 \mathrm{mg}$ glucose/ $\mathrm{kg}$ body weight; $p<0.001$ ) (Fig. 3). Since there was no statistical difference in post-atropine RIST index between standard-fed and any sucrose-fed group (89.6 \pm 4.1 [standard-fed] and $78.9 \pm 10.6$ [2 weeks sucrose-fed], $61.0 \pm 10.1$ [6 weeks sucrose-fed] and $88.8 \pm 6.7$ [ 9 weeks sucrose-fed] $\mathrm{mg}$ glucose $/ \mathrm{kg}$ body weight), the decrease of total insulin sensitivity was due to lower HPN-dependent component in all sucrose-fed groups $(102.7 \pm 6.9$ [standard-fed] vs $28.5 \pm$ 7.6 [2 weeks sucrose-fed], $35.3 \pm 21.4$ [6 weeks sucrose-fed] and $17.9 \pm 5.4$ [9 weeks sucrose-fed] $\mathrm{mg}$ glucose $/ \mathrm{kg}$ body weight; $p<0.001)$. Accordingly, the contribution of the HPN-dependent component to total insulin sensitivity was lower in all sucrose-fed groups than in the standard-fed group $(52.5 \pm 2.6 \%$ [standard-fed] vs $26.4 \pm 6.2 \%$ [ 2 weeks sucrose-fed], $28.8 \pm 13.8 \%$ [6 weeks sucrose-fed] and $17.9 \pm$ 


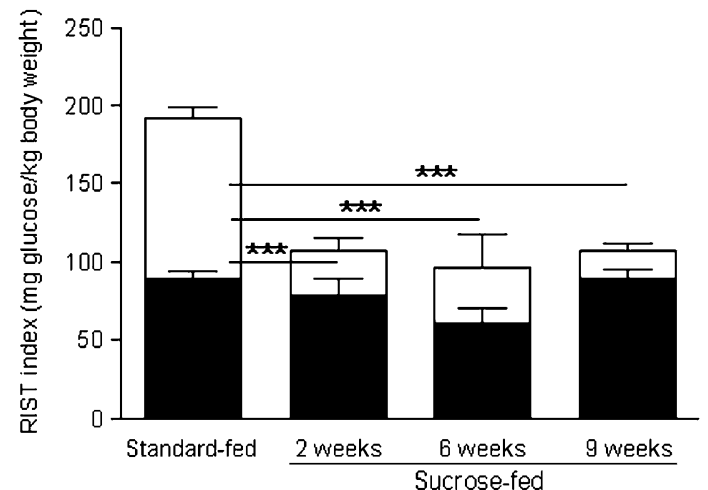

Fig. 3 RIST indexes for the HPN-independent (filled) and HPNdependent (open) components of insulin action of Sprague-Dawley rats on a sucrose diet for different periods. The sum of both components represents the control RIST index. Insulin sensitivity was significantly higher for the standard-fed group than for any sucrosefed group. The HPN-dependent component accounted for this variation $(* * * p<0.001)$

5.4\% [9 weeks sucrose-fed]; $p<0.01, p<0.05$ and $p<0.001$ respectively).

Effect of a liquid vs solid high-sucrose diet in SpragueDawley rats Total body weight and blood pressure were similar in all groups (data not shown). The liquid-sucrose-fed group had a higher basal glycaemia than both standard-fed and solid-sucrose-fed groups (7.91 \pm 0.49 [liquid-sucrose-fed] vs $6.37 \pm 0.42$ [standard-fed] and $6.21 \pm$ 0.16 [solid-sucrose-fed] mmol glucose $/ 1 ; p<0.05$ ).

Both solid- and liquid-sucrose-fed groups had similarly lower total insulin sensitivity than the standard-fed group (185.6 \pm 8.6 [standard-fed, $n=9$ ] vs $88.2 \pm 14.6$ [solid-sucrose-fed, $n=6$ ] and 106.6 \pm 8.4 [liquid-sucrose-fed, $n=11$ ] $\mathrm{mg}$ glucose $/ \mathrm{kg}$ body weight; $p<0.001$ and $p<0.05$ respectively) (Fig. 4). After atropine administration, the RIST indexes obtained were similar in all three groups $(97.9 \pm 2.9$ [standard-fed], $76.5 \pm 14.1$ [solid-sucrose-fed] and $88.8 \pm 6.7$ [liquid-sucrose-fed] $\mathrm{mg}$ glucose $/ \mathrm{kg}$ body weight). The HPN-

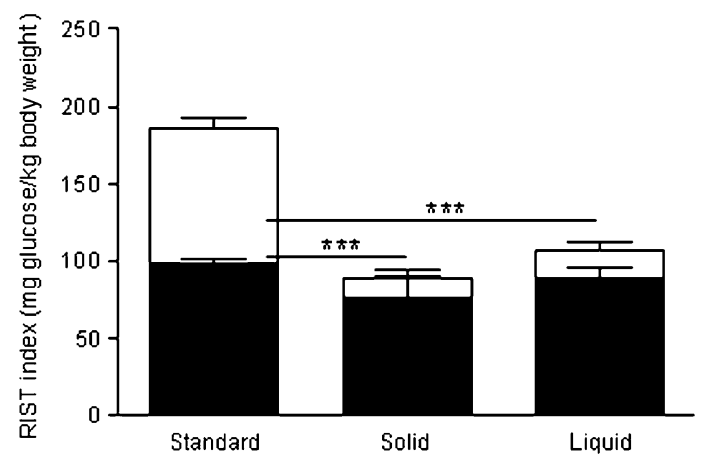

Fig. 4 RIST indexes for the HPN-independent (filled) and HPNdependent (open) components of insulin action of Sprague-Dawley rats fed either a standard diet or a liquid or solid sucrose diet. The sum of both components represents the control RIST index. Insulin sensitivity was significantly higher for the standard diet than for any of the high-sucrose-fed groups. The HPN-dependent component accounted for this difference $(* * * p<0.001)$ dependent component was lower in both sucrose-fed groups than in the standard-fed group (87.6 7.3 [standard-fed] vs $12.3 \pm 4.7$ [solid-sucrose-fed] and $17.9 \pm 5.4$ [liquid-sucrosefed] $\mathrm{mg}$ glucose/kg body weight; $p<0.001$ ); thus the contribution of the HPN-dependent component was also lower in sucrose-fed animals than in the standard-fed group (46.7 \pm $2.0 \%$ [standard-fed] vs $13.3 \pm 7.3 \%$ [solid-sucrose-fed] and $15.3 \pm 4.3 \%$ [liquid-sucrose-fed]; $p<0.001$ ).

Some of these results were previously reported by us in abstract form [18].

\section{Discussion}

Our study aimed to relate the HISS hypothesis, a pathway dependent upon the HPN modulation of whole-body insulin-stimulated glucose uptake, to the insulin resistance caused by a high-sucrose diet.

The Wistar rats showed higher insulin-stimulated glucose uptake than the Sprague-Dawley rats. However, the partial contribution of each component was similar in the two strains for both standard-fed and sucrose-fed animals.

Our results show that the insulin resistance present in rats subjected to a high-sucrose diet is indeed due to an impairment of the HPN-dependent component of insulin action, which is already observed after 2 weeks of the diet. There were no differences in results between the two forms of sucrose feeding (solid vs liquid).

This suggests that the HPN-dependent component, and subsequently HISS action, is easily impaired by a highsucrose diet, with the HISS-independent component of insulin action remaining unimpaired, at least for the first 9 weeks of the diet.

\section{Methodological considerations}

In our study, the quantification of whole-body insulin sensitivity was carried out using a modified euglycaemic clamp known as the RIST (rapid insulin sensitivity test) [19]. Designed to avoid the counter-regulatory responses to the hypoglycaemia that follow the administration of an insulin bolus, the RIST has reproducibility in four consecutive tests in anaesthetised rats [19], allowing us to measure control and post-atropine insulin sensitivity in the same animal. RIST assessment is comparable to the insulin tolerance test and has been shown to be more sensitive in detecting the HISS-dependent component of insulin action than the hyperinsulinaemic-euglycaemic clamp [20]. Since HISS has been shown to decrease in the fasting state [21], it is essential to assess whole-body insulin sensitivity in the fed state.

Our experiments were performed with the animals under anaesthesia. Although pentobarbital anaesthesia has been shown to produce insulin resistance [22], previous studies done with the RIST showed no difference in glucose uptake between anaesthetised and conscious rats [23]. A possible explanation is that maintaining euthermia, as we did using a heating pad and a rectal probe, prevents the anaesthesiainduced alterations in glucose metabolism [24]. 
The administration of atropine $(3 \mathrm{mg} / \mathrm{kg})$ does not interfere with hepatic glucose output inhibition by insulin and does not alter basal glycaemia or insulinaemia [2, 4]. A dose-response curve produced by our group [25] shows that the $\mathrm{ED}_{50}$ of atropine administered intraportally was lower than that administered intravenously, establishing that the relevant affected receptors are located in the liver [25]. Since intraportal administration of atropine did not show, at the given dose, a statistically different effect from intravenous administration $[19,25]$, we chose the latter to minimise the invasive surgery required.

Insulin resistance in sucrose-fed animals

Effect of a liquid high-sucrose diet on Wistar and SpragueDawley rats In sucrose-fed Wistar and Sprague-Dawley rats, we observed a considerable decrease in postprandial total insulin-stimulated glucose uptake in relation to the standard-fed rats. This result, shown by comparing standardfed and sucrose-fed control RIST indexes for each rat strain, supports the findings of other authors. Sucrose-enriched diets have been reported to lead to skeletal muscle insulin resistance [13-16]. However, none of the previous studies took into consideration that insulin-stimulated glucose uptake consists of two discernible components, one of which is modulated by the hepatic parasympathetic nerves [1].

To evaluate the relevance of the HPN-dependent component of insulin action in this animal model of insulin resistance, we used atropine, a muscarinic receptor antagonist that has been found to elicit, in normal-fed animals, a similar decrease in whole-body insulin-stimulated glucose uptake to that elicited by the surgical ablation of the hepatic parasympathetic nerves [26]. Atropine only decreased insulin sensitivity in the standard-fed rats. Sucrose-fed rats showed similar pre- and post-atropine RIST indexes. Furthermore, the insulin sensitivity of post-atropine standard-fed rats was not statistically different from that obtained for pre- and post-atropine sucrose-fed rats, thus showing that the postprandial whole-body insulin resistance presented by sucrose-fed rats is entirely derived from the impairment of the HPN-dependent component of insulin action, and also that this component is essentially absent in the sucrose-fed rats.

By examining the mean dynamic profiles of the RISTs and their characteristics we draw similar conclusions, even when more details are examined. The dynamic profile of the control RIST of sucrose-fed rats showed a lower peak magnitude and an earlier offset of action than the control RIST in standard-fed rats. Considering that the HPNindependent component (post-atropine) dynamic profile of standard-fed and sucrose-fed rats is similar, the detrimental effect of the high-sucrose diet is entirely accounted for by the lower peak magnitude and duration seen in the HPNdependent dynamic profile of sucrose-fed rats.

Effect of the duration of a liquid high-sucrose diet in Sprague-Dawley rats The present study also shows that insulin resistance caused by HPN impairment is fully expressed after only 2 weeks of the high-sucrose diet. The sucrose-fed groups showed a higher weight gain in relation to the standard-fed group with the longest diet duration (9 weeks). We can therefore exclude weight gain as a confounding factor since there was no additional increase of insulin resistance in this group. Likewise, as all groups showed similar blood pressures, we can exclude the interference of hypertension.

In addition, since basal hyperglycaemia was only observed in the rats exposed to the sucrose supplement for 9 weeks, we conclude that the impairment of the HISSdependent component is not the result of high blood glucose levels, because sucrose-fed rats exposed for 2 or 6 weeks were similarly insulin resistant, despite having normoglycaemia.

Effect of a liquid vs solid high-sucrose diet in SpragueDawley rats We did not find any difference in insulin sensitivity between the two forms of sucrose feeding (solid vs liquid). Thus, we can only conclude that this is not, at the given concentrations, a factor that influences the degree of insulin resistance.

Hepatic parasympathetic nerves dysfunction and the development of insulin resistance

The present findings highlight the importance, in the study of insulin resistance in high-sucrose-fed animals, of recent reports of decreased parasympathetic vagal activity in highfructose-fed rats [11]. Absorbed sucrose is degraded into glucose and fructose, and fructose has been found to be the moiety responsible for sucrose-induced insulin resistance [27]. Thus, we can suggest that a high-sucrose diet, perhaps through the metabolic action of its fructose moiety, leads to HPN-dependent insulin resistance.

Recently, an increasing emphasis is being placed on a causal effect of an autonomic imbalance in the metabolic syndrome [28]. In this imbalance, brought about by environmental factors, the decrease of parasympathetic activity seems to be the determining factor in the development of insulin resistance and type 2 diabetes mellitus [29]. Our study is an example of how an environmental alteration, such as diet composition, can lead to insulin resistance through an impairment of hepatic parasympathetic activity. Moreover, preliminary studies in our laboratory show that the impairment of the HPN-dependent component of insulin action is also involved in the development of insulin resistance in conditions commonly included in the metabolic syndrome, such as obesity [30] and essential hypertension [31].

It has been reported that food intake enhances the parasympathetic tone [32]. It is thus essential that the RIST is performed in the immediate postprandial state to properly quantify the HPN-dependent component of insulin action. Indeed, fasting has been shown to inhibit the HPN-dependent component of insulin action while having no effect on the HPN-independent component [21].

Our present results are consistent with the mechanism first described by one of us [1] in which postprandial hepatic 
parasympathetic activity leads to the release of a hepatic humoral factor, tentatively named HISS (hepatic insulinsensitising substance), which sensitises the skeletal muscle to insulin-stimulated glucose uptake. HISS-dependent insulin resistance has been found in liver diseases, fetal alcohol exposure, acute stress and ageing [33]. Moreover, sucrose-induced insulin resistance in skeletal muscle has been related to the impairment of a humoral factor, which would explain why only in vivo and not in vitro studies show skeletal muscle insulin resistance in sucrose-fed rats when compared with starch-fed rats [15].

Other studies by our groups [34, 35] have previously uncovered other steps of the hepatic pathway that lead to HISS release. According to these studies, following a meal, the activation of the hepatic parasympathetic nerves leads to the release of acetylcholine, which binds to muscarinic receptors in the liver. This promotes the production of hepatic nitric oxide and the release of HISS. Hepatic glutathione, which is decreased in the fasting state and rapidly replenished in the postprandial state, is also essential to the HISS pathway. Interestingly, hepatic glutathione and nitric oxide could also play a role in HISS-dependent insulin resistance in sucrose- or fructose-fed animals because a high-sucrose diet alters hepatic glutathione enzyme activity [36] and nitric oxide donors ameliorate fructose-induced insulin resistance [37].

Further studies using the sucrose-fed animal model, which is free of the confounding effects of weight gain and hypertension, will give us more information about the effects of a high-sucrose diet on the HISS pathway, furthering our knowledge of possible therapeutic interventions to prevent or ameliorate HISS-dependent insulin resistance. Interestingly, exercise training, which increases vagal activity [38], is capable of preventing sucrose-diet-induced insulin resistance [39].

\section{Conclusions}

Our data show that a high-sucrose diet leads to insulin resistance by rapid impairment of the HISS pathway. This impairment, due to a decreased activity of the HPN which is also observed in other pathological conditions related to insulin resistance, the metabolic syndrome and type 2 diabetes mellitus, illustrates the dramatic deleterious effect of a disruption of the HISS pathway on the regulation of insulinstimulated glucose uptake.

Acknowledgements We thank P. F. Costa and A. I. Santos for helpful discussions.

This study was supported by Foundation of Science and Technology (FCT) grants POCTI/SAL/14009/1998 and POCTI/NSE/42397/2001 and by the Portuguese Diabetes Association (APDP). Funding was also provided via a Canadian Institutes of Health Research IHRT Group Grant.

Duality of interest

W. W. Lautt is affiliated with DiaMedica (Winnipeg, Manitoba, Canada), the licensor of technology to diagnose and treat insulin resistance through the HISS pathway.

\section{References}

1. Lautt WW (1999) The HISS story overview: a novel hepatic neurohumoral regulation of peripheral insulin sensitivity in health and diabetes. Can J Physiol Pharmacol 77:553-562

2. Xie H, Lautt WW (1995) Induction of insulin resistance by cholinergic blockade with atropine in the cat. J Auton Pharmacol 15:361-369

3. Latour MG, Lautt WW (2002) Insulin sensitivity regulated by feeding in the conscious unrestrained rat. Can J Physiol Pharmacol $80: 8-12$

4. Xie H, Lautt WW (1996) Insulin resistance caused by hepatic cholinergic interruption and reversed by acetylcholine administration. Am J Physiol 271:E587-E592

5. Moore MC, Satake S, Baranowski B, Hsieh P, Neal DW, Cherrington AD (2002) Effect of hepatic denervation on peripheral insulin sensitivity in conscious dogs. Am J Physiol 282:E286-E296

6. Petersen KF, Tygstrup N (1994) A liver factor increasing glucose uptake in rat hindquarters. J Hepatol 20:461-465

7. Hendrickse MT, Triger DR (1993) Autonomic dysfunction in chronic liver disease. Clinic Auton Res 3:227-231

8. Gentile S, Marmo R, Peduto A, Montella F, Coltorti M (1994) Autonomic neuropathy in liver cirrhosis: relationship with alcoholic aetiology and severity of the disease. Ital J Gastroenterol 26:53-58

9. Fleisher LA, Fleckenstein JF, Frank SM, Thuluvah PJ (2000) Heart rate variability as a predictor of autonomic dysfunction in patients awaiting liver transplantation. Dig Dis Sci 45:340-344

10. Valensi P, Nguyen TN, Idriss S et al (1998) Influence of parasympathetic dysfunction and hyperinsulinemia on the hemodynamic response to an isometric exercise in non-insulin-dependent diabetic patients. Metabolism 47:934-939

11. Miller AW, Sims JJ, Canavan A, Hsu T, Ujhelyi MR (1999) Impaired vagal reflex activity in insulin-resistant rats. J Cardiovasc Pharmacol 33:698-702

12. Carnethon MR, Jacobs DR Jr (2003) Influence of autonomic nervous system dysfunction on the development of type 2 diabetes. Diabetes Care 26:3035-3041

13. Pagliassotti MJ, Shahrokhi KA, Moscarello M (1994) Involvement of liver and skeletal muscle in sucrose-induced insulin resistance: dose-response studies. Am J Physiol 266:R1637-R1644

14. Hulman S, Falkner B (1994) The effect of excess dietary sucrose on growth, blood pressure, and metabolism in developing Sprague-Dawley rats. Pediatr Res 36:95-101

15. Kim JY, Nolte LA, Hansen PA, Han DH, Kawanaka K, Holloszy JO (1999) Insulin resistance of muscle glucose transport in male and female rats fed a high-sucrose diet. Am J Physiol 276: R665-R672

16. D'Alessandro ME, Chicco A, Karabatas L, Lombardo YB (2000) Role of skeletal muscle on impaired insulin sensitivity in rats fed a sucrose-rich diet: effect of moderate levels of dietary fish oil. J Nutr Biochem 11:273-280

17. Gaudreault N, Santuré M, Pitre M, Nadeau A, Marette A, Bachelard H (2001) Effects of insulin on regional blood flow and glucose uptake in Wistar and Sprague-Dawley rats. Metabolism 50:65-73

18. Ribeiro RT, Duarte-Ramos F, Macedo MP (2001) The action of hepatic insulin sensitising substance is decreased in rats on a high-sucrose diet. Proc West Pharmacol Soc 44:31-32

19. Lautt WW, Wang X, Sadri P, Legare DJ, Macedo MP (1998) Rapid insulin sensitivity test (RIST). Can J Physiol Pharm 76:1-7

20. Reid MAG, Latour MG, Legare DJ, Rong N, Lautt WW (2002) Comparison of the rapid insulin sensitivity test (RIST), the insulin tolerance test (ITT), and the hyperinsulinemic euglycemic clamp (HIEC) to measure insulin action in rats. Can J Physiol Pharm 80:811-818

21. Lautt WW, Macedo MP, Sadri P, Takayama S, Duarte-Ramos F, Legare J (2001) Hepatic parasympathetic (HISS) control of insulin sensitivity determined by feeding and fasting. Am J Physiol 281:G29-G36 
22. Pénicaud L, Ferré P, Kande J, Leturque A, Issad T, Girard J (1987) Effect of anesthesia on glucose production and utilization in rats. Am J Physiol 252:E365-E369

23. Latour MG, Lautt WW (2002) Insulin sensitivity regulated by feeding in the conscious unrestrained rat. Can J Physiol Pharm 80:8-12

24. Lang CH, Bagby GJ, Hargrove DM, Hyde PM, Spitzer JJ (1987) Alterations in glucose kinetics induced by pentobarbital anesthesia. Am J Physiol 253:E657-E663

25. Takayama S, Legare DJ, Lautt WW (2000) Dose-related atropine-induced insulin resistance: comparing intraportal versus intravenous administration. Proc West Pharmacol Soc 43:33-34

26. Xie H, Lautt WW (1994) Insulin resistance produced by hepatic denervation or muscarinic cholinergic blockade. Proc West Pharmacol Soc 37:39-40

27. Thresher JS, Podolin DA, Wei Y, Mazzeo RS, Pagliassotti MJ (2000) Comparison of the effects of sucrose and fructose on insulin action and glucose tolerance. Am J Physiol 279:R1334R1340

28. Kreier F, Yilmaz A, Kalsbeek A et al (2003) Hypothesis: shifting the equilibrium from activity to food leads to autonomic unbalance and the metabolic syndrome. Diabetes 52:2652-2656

29. Lindmark S, Wiklund U, Bjerle P, Eriksson JW (2003) Does the autonomic nervous system play a role in the development of insulin resistance? A study on heart rate variability in firstdegree relatives of type 2 diabetes patients and control subjects. Diabet Med 20:399-405

30. Ribeiro RT, Duarte-Ramos F, Macedo MP (2001) The fatty Zucker rat fa/fa shows a dysfunction of the HISS-dependent and -independent components of insulin action. Proc West Pharmacol Soc 44:29-30
31. Ribeiro RT, Duarte-Ramos F, Macedo MP (2001) Effect of hepatic insulin sensitizing substance in the spontaneously hypertensive rat. Proc West Pharmacol Soc 44:27-28

32. Uijtdehaage SH, Stern RM, Koch KL (1992) Effects of eating on vection-induced motion sickness, cardiac vagal tone, and gastric myoelectric activity. Psychophysiology 29:193-201

33. Lautt WW (2004) A new paradigm for diabetes and obesity: the hepatic insulin sensitizing substance (HISS) hypothesis. J Pharmacol Sci 95:9-17

34. Guarino MP, Afonso RA, Raimundo N, Raposo JF, Macedo MP (2003) Hepatic glutathione and nitric oxide are critical for hepatic insulin-sensitizing substance action. Am J Physiol 284: G588-G594

35. Sadri P, Lautt WW (1999) Blockade of hepatic nitric oxide synthase causes insulin resistance. Am J Physiol 277:G101G108

36. Peters LP, Teel RW (2003) Effects of high sucrose diet on body and liver weight and hepatic enzyme content and activity in the rat. In vivo 17:61-65

37. Oshida Y, Tachi Y, Morishita Y et al (2000) Nitric oxide decreases insulin resistance induced by high-fructose feeding. Horm Metab Res 32:339-342

38. De Angelis K, Wichi RB, Jesus WRA et al (2004) Exercise training changes autonomic cardiovascular balance in mice. J Appl Physiol 96:2174-2178

39. Wright DW, Hansen RI, Mondon CE, Reaven GM (1983) Sucrose-induced insulin resistance in the rat: modulation by exercise and diet. Am J Clin Nutr 38:873-883 